\title{
Diabetes Related Distress and Depression: An Emerging Threat to Human Health
}

\author{
Chidambaram Ramasamy* \\ Department of Prosthodontics, Faculty of Dentistry, AIMST University, Bedong, Kedah Darul Aman, MALAYSIA. \\ Correspondence
}

Chidambaram Ramasamy, Department of Prosthodontics, Department of Advance Conservative Dentistry, Faculty of Dentistry, AlMST University, Jalan Bedong- Semeling, 08100, Bedong, Kedah Darul Aman, MALAYSIA.

Phone:+60-016-4724370

Email: dr.ramasamyc@gmail.com

DOI: 10.5530/jyp.2018.10.57

Diabetes is a disease which is intricate to manage. According to the International Diabetes Federation (IDF), more than 415 million people have diabetes in the world, and it is predicted that the number will rise to 642 million people with diabetes by $2040 .{ }^{1}$ Treatment consists of lifestyle adaptations often combined with medication to control blood glucose levels. Despite available treatment, diabetes is often associated with complications and co-morbidities which increase the complexity of disease management even further. Complications and comorbidities are of particular concern in diabetes. Diabetes is usually accompanied by many comorbidities such as hypertension, dyslipidemia, and cardiovascular diseases. ${ }^{2}$ Uncontrolled diabetes mellitus also leads to the development of many micro-vascular complications such as nephropathy, retinopathy, and neuropathy as well as macro-vascular complications such as coronary artery disease, peripheral arterial disease and stroke. ${ }^{3}$ These complications and co-morbidities, together with the emotional stress associated with the diagnosis of diabetes, had been linked to the development of depression and anxiety among diabetic patients. ${ }^{4}$

Depression and diabetes are chronic disease states with the potential to impact many aspects of life. Patients with type 1 diabetes mellitus (T1DM) or type 2 diabetes mellitus (T2DM) may become burdened with the self-care challenges associated with a diabetes diagnosis, and this psychological stress may precipitate depression. ${ }^{5}$ On the other side, depression may serve as a risk factor for patients with T2DM. This may be due to lifestyle habits associated with depression. These lifestyle habits may include poor health behaviours such as smoking, high-fat diet, and excessive alcohol intake, all of which contribute to an increased risk of diabetes. ${ }^{6}$ A large literature that seems to have grown exponentially over the last decade demonstrates increased prevalence of depression in diabetes patients as compared to those without diabetes..$^{7-9}$ Compared to people without diabetes, depression prevalence rates are over three times higher in people with T1DM and twice as high in patients with T2DM. ${ }^{9}$ Traditionally it is believed that the link between depression and diabetes are related to the burden of chronic disease management. ${ }^{10}$ Recent evidences suggest that the relationship between diabetes and depression is likely to be bi-directional. ${ }^{11}$. The implication of this finding is that a diagnosis with diabetes mellitus increases the risk of depression and in contrast, depression is a well-established risk factor for diabetes in nondiabetic individuals. Furthermore, depression could act as a contributor to, and an accelerator of, the complications of diabetes. Coping with such health-threats may seem burdensome to patients and might affect them mentally and lead to a psycho-social problem.

Patients with diabetes can also develop a disorder that mimics symptoms of depression called diabetes-related distress (DRD), which is associated with the challenges of living with diabetes. DRD affects an individual's problem solving skill which is required to carry out diabetes self-care and this may result in poorer self-care practices, and ultimately poorer glycemic control. Depression and distress in T2DM are associated with greater morbidity, mortality and health-care costs. ${ }^{12}$ It is projected that by the year 2020, depression will be the second leading contributor to the global burden of disease. ${ }^{13}$ Despite the seeming efforts of health care professionals/organisations and anti-depression programmes, depression is often failed to be noticed in the health care system as an important illness to treat. Nevertheless, depression stills remain rather under-diagnosed and undertreated. Though the bi-directional link between DRD and depression is alarming, it does provide a new opportunity to re-consider our strategy. In this climate of health care and cost containment, identifying depression with early treatment is essential in preventing the associated physical diseases not limited to stroke and cardiovascular disease. From the patient's perspective, depression and diabetes are not discussed much. Patients on the other hand find it hard to empathize and like to talk to someone who is semi-professional and often has more time to listen. A client could be experiencing a chronic or life-changing illness, have a trauma from a critical event, have pre-existing mental illness, be dealing with significant health or personal loss, be using substances, or experiencing a depression. At some point we will treat a client who may have a diagnosed depression, appear depressed, or have thoughts of suicide. ${ }^{14}$ When dealing with suspected/ undiagnosed depression in diabetes patients, however, the strain resulting from interaction with the patients should not be underestimated. Henceforth the worker should have basic skills in recognising poor mood and have a list of useful questions to ask in a crisis. Most importantly one should be able to distinguish depression from DRD in patients with diabetes because this distinction affects how patients are medically managed. The American Diabetes Association (ADA) guidelines recommend psychological and social assessment as part of diabetes management, including screening for depression and DRD. The ADA guidelines recommend that patients with diabetes be routinely screened for psychosocial problems (e.g., de-

This is an open access article distributed under the terms of the Creative Commons Attribution-NonCommercial-ShareAlike 4.0 License, which allows others to remix, tweak, and build upon the work non-commercially, as long as the author is credited and the new creations are licensed under the identical terms. 
pression) with the Patient Health Questionnaire (PHQ)-2, PHQ-9 (as follow-up if the PHQ-2 is positive), or another validated screening tool. ${ }^{15}$ Health-care providers should be encouraged to use both screening tools on patients with suspected depression or diabetes distress during initial and follow-up visits to monitor for changes in depressive symptoms and to monitor the patients' perceived emotional well-being over time. It is also important for the provider to include a thorough history and physical examination in addition to the self-report questionnaire, as many issues may be discovered that the screening tool would not have revealed. Additionally, studies suggest that treatment of depression may be associated with normalization of blood glucose. ${ }^{7}$ Treatment options include pharmacotherapy, psychotherapy (most commonly cognitivebehavioural therapy [CBT]), or a combination of the two. CBT, a method of reframing negative thoughts is a widely documented, empirically validated type of psychotherapy and effective in lessening the depressive symptoms ${ }^{15}$ Furthermore, beneficial effects on glycemic control occurs when CBT includes diabetes self-management education. With sufficient dosing, antidepressants can ameliorate depressive symptoms in diabetes patients at a level comparable to that seen in the general population. ${ }^{10}$

Even though most of the patients dismiss the depression as a matter of concern, mental health of clients is everyone's responsibility, especially those working in health care. Since health- care professionals play a vital role in the holistic care of the patient, ensuring that they are skilled and trained efficiently helps to reduce the total cost of care, improve the quality and accessibility to health-care service. Most importantly, they should know their local crisis and supportive mental health services, create links with them, have ongoing professional education and protocols for managing clients at-risk, and are acutely aware of their role and limitations. As advocates for patients, health care professionals often are the first to recognize problems and frequently serve as safety nets. The extended scope role for health-care professionals is one strategy that could be undertaken by health-care system to meet this new demand. For instance, the pharmacist can also provide education and medication management, empower patients to develop appropriate coping skills, and serve as a consistent monitoring source between physician visits. In order to maximize the benefits of team-based care, medical practitioners should recognize the larger role that allied health professionals' play and support them as increasingly valuable team members. ${ }^{16}$ Collaborative care should be combined with treatment interventions, education, and support to optimize health outcomes in patients. It is the need of hour that we seriously take advantage of the growing multidisciplinary approach in today's internet world to improvise the well-being of our patients. Given the high prevalence of depression and DRD, routine screening of patients with diabetes should be encouraged to optimise mental health and improve quality of life.

\section{REFERENCES}

1. IDF Diabetes Atlas: Global estimates for the prevalence of diabetes for 2015 and 2040. Diabetes Res Clin Pract. 2017;128:40-50.

2. Dagogo-Jack S. Metabolomic prediction of diabetes and cardiovascular risk. Med Princ Pract. 2012;21(5):401-403.

3. Michael J Flower. Microvascular and macrovascular complications of diabetes. Clinical Diabetes. 2008;26(2):77-82.

4. Gonzalez JS, Esbitt SA, Schneider HE, Osborne PJ, Kupperman EG. Psychological issues in adults with type 2 diabetes; in Pagoto $S$ (eds): Psychological CoMorbidities of Physical Illness: A Behavioral Medicine Perspective. New York, Springer Science Business Media LLC. 2011:73-121.

5. Berge LI, Riise T. Comorbidity between type 2 diabetes and depression in the adult population: directions of the association and its possible pathophysiological mechanisms. Int J Endocrinol. 2015, Article ID 164760, 7 pages

6. Campayo A, Gómez-Biel CH, Lobo A. Diabetes and depression. Curr Psychiatry Rep. $2011 ; 13: 26-30$

7. Anderson R, Freedland K, Clouse R, Lustman P. The prevalence of comorbid depression in adults with diabetes: a meta-analysis. Diabetes Care. 2001;24:1069 78.

8. Ali S, Stone MA, Peters JL, Davies MJ, Khunti K. The prevalence of co-morbid depression in adults with type 2 diabetes: a systematic review and meta-analysis. Diabet Med. 2006;23(11):1165-73.

9. Kathryn EK. Diabetes distress or major depressive disorder? a practical approach to diagnosing and treating psychological comorbidities of diabetes. Diabetes Ther. 2017;8(1):1-7.

10. Holt RI, De Groot M, Golden SH. Diabetes and depression. Curr Diab Rep. 2014;14(6):1-9

11. Alzoubi, A, Abunaser R, Khassawneh A, Alfaqih M, Khasawneh A, Abdo N. The bidirectional relationship between diabetes and depression: a literature review. Korean J of Fam Medi. 2018;39(3):137-46.

12. Egede LE, Walker RJ, Bishu K, Dismuke CE. Trends in costs of depression in adults with diabetes in the United States: medical expenditure panel survey. 2004-2011. J Gen Intern Med. 2016;31:615-22.

13. Vigo D, Thornicroft G, Atun R. Estimating the true global burden of mental illness. Lancet Psychiatry. 2016;3(2):171-8.

14. Donley E. Suicide risk of your client: initial identification and management for the allied health professional. J Allied Health. 2013;42(1):56-61.

15. American Diabetes Association standards of medical care in diabetes-2016. Diabetes Care. 2016;39 (suppl 1):S1-S112.

16. Sheridan B, Chien AT, Peters AS, Rosenthal MB, Brooks JV, Singer SJ. Teambased primary care: The medical assistant perspective. Health Care Manage Rev. 2018;43(2):115-25. 